\title{
Influence of radiation treatment technique on outcome and toxicity in anal cancer
}

\author{
Elisha T. Fredman ${ }^{1} \cdot$ May Abdel-Wahab ${ }^{2}$ - Aryavarta M.S. Kumar ${ }^{1,3}$
}

Received: 13 July 2017 / Accepted: 9 August 2017 / Published online: 22 August 2017

(C) The Author(s) 2017. This article is an open access publication

\begin{abstract}
Objective Intensity-modulated radiation therapy (IMRT) has largely supplanted three-dimensional conformal radiation (3D-CRT) for definitive anal cancer treatment due to decreased toxicity and potentially improved outcomes. Convincing data demonstrating its advantages, however, remain limited. We compared outcomes and toxicity with concurrent chemotherapy and IMRT vs 3D-CRT for anal cancer. Methods We performed a single-institution retrospective review of patients treated with IMRT or 3D-CRT as part of definitive mitomycin-C/5-fluorouricil-based chemoradiation for anal cancer from January 2003 to December 2012.

Results One hundred sixty-five patients were included, with 61 and 104 receiving IMRT and 3D-CRT, respectively. Overall, $92.7 \%$ had squamous cell carcinoma. The mean initial pelvic dose was 48.3 and 44 Gy for IMRT and 3D-CRT, respectively. Complete response, partial response, and disease progression rates were similar (IMRT 83.6, 8.2, 8.2\%; 3DCRT 85.6, 6.7, 7.7\%; $p=0.608, p=0.728, p=0.729$ ). There was no significant difference in overall survival $(p=0.971)$, event-free survival $(p=0.900)$, or local or distant recurrence rates $(p=0.118, p=0.373)$. IMRT caused
\end{abstract}

Elisha T. Fredman

elisha.fredman@uhhospitals.org

1 Department of Radiation Oncology, Seidman Cancer Center, University Hospitals Cleveland Medical Center, 11100 Euclid Ave, Cleveland, OH 44106, USA

2 Department of Nuclear Sciences and Applications, International Atomic Energy Agency's (IAEA) Division of Human Health, Vienna, Austria

3 University Hospitals Parma Seidman Cancer Center, Parma, OH, USA significantly less acute grade $1-2$ incontinence $(p=0.035)$, grade $3-4$ pain $(p=0.033)$, and fatigue $(p=0.030)$. IMRT patients had significantly fewer chronic post-treatment toxicities ( $p=0.008$ ), outperforming 3D-CRT in six of eight toxicities reviewed. Though total treatment length was comparable (43.6 and 44.5 days), IMRT recipients had fewer ( 27.9 vs $41.3 \%$ of patients, $p=0.89$ ), shorter treatment breaks (mean 2.9 vs 4.1 days, $p=0.229$ ).

Conclusion This report represents the largest series directly comparing concurrent chemotherapy with IMRT vs 3D-CRT for definitive treatment of anal cancer. IMRT significantly reduced acute and post-treatment toxicities and allowed for safe and effective pelvic dose escalation.

Keywords Anal cancer $\cdot$ Radiation $\cdot$ IMRT $\cdot 3 \mathrm{D} \cdot$ Toxicity

\section{Introduction}

Eight thousand eighty people in the USA are expected to be diagnosed with anal cancer in 2016, resulting in 1080 deaths [1]. Despite comprising only $1.5 \%$ of all gastrointestinal tumors, the rates of diagnosis have been increasing in the USA and globally $[2,3]$. The current standard of care is definitive chemoradiation, with abdominoperineal resection (APR) reserved for salvage therapy $[4,5]$.

Advancements in radiation treatment over time have attempted to better optimize its delivery to potentially reduce toxicity and allow for maximal dose escalation. Historically, two-dimensional techniques based on surface anatomy and bony landmarks were used to deliver radiation for anal cancer. Until recently, three-dimensional conformal radiation (3DCRT) was the most commonly utilized treatment modality, incorporating $\mathrm{CT}$ imaging data to better identify the intended target. Intensity-modulated radiation therapy (IMRT) 
followed, which can be used to design still more conformal radiation fields. By modulating the intensity of each beam delivered, a dose can be designed to target the concavities and convexities of a tumor volume, thereby further reducing dose to adjacent tissues.

Within the last few years, IMRT has been shown to be more efficacious and less toxic than traditional 3D-CRT for multiple disease sites [6-9]. Smaller case series have reported similar advantages of IMRT in the context of anal cancer, but conclusive data remain limited [10-15]. Though these studies present survival and toxicity outcomes, even fewer are comparative in nature $[12,13]$. Furthermore, a wide range of results have been reported thus far, from a significant survival benefit with IMRT to more modest results of decreased toxicities and fewer treatment breaks $[11,12,15,16]$. Since IMRT is both substantially more expensive and technically demanding, it is critical to rigorously evaluate the scope of its benefit. We therefore present a comparison of patients treated for anal squamous cell carcinoma with 3D-CRT vs IMRT, with an emphasis on acute toxicity, chronic posttreatment sequelae, and clinical outcomes.

\section{Methods}

Internal Review Board approval was obtained to perform a retrospective review of all anal cancer patients evaluated at our institution between January 2003 and December 2012. Patients had biopsy-confirmed invasive anal cancer treated with definitive chemoradiation using either 3D-CRT or IMRT, including patients with regional nodal involvement. Patients were excluded if they had distant metastases or if treatment intent was palliative. Of the 249 patients initially identified, 165 were eligible. All patients underwent complete staging using contrastenhanced CT, MRI, or FDG-PET.

Pertinent demographic information and tumor characteristics were collected based on previously identified risk factors for the development of anal cancer [17]. Tumor factors included histological type, grade, AJCC stage (7th edition), and distance from the anal verge measured on imaging and exam. Acute toxicities during treatment were collected from weekly on-treatment and radiation completion notes, while post treatment, late toxicity were collected from radiation and medical oncology follow-up documentation.

\section{Chemotherapy}

Patients received 2 cycles of concurrent infusional 5fluorouracil (5-FU)-based cytotoxic chemotherapy. In the majority of instances, treatment entailed combination 5-FU and bolus mitomycin-C (MMC) per the Nigro protocol [18].
Based on practice variability among medical oncologists, $10.9 \%$ of patients received 5-FU alone, 5-FU/cisplatin, oral Xeloda, 5-FU/cisplatin/vinblastine, or an etoposide-based treatment.

\section{Radiation therapy}

All patients underwent CT simulation, either prone with a belly board or supine. Non-IMRT radiotherapy planning followed classic two-dimensional radiotherapy borders for mini-pelvic fields, cone downs, and tumor boosts [19]. Electrons were used to supplement dose to the inguinal lymph nodes. IMRT contours and dose constraints were according to RTOG 05-29 [20]. Briefly, contours including the gross tumor, and elective lymph node regions (inguinal, internal iliac, external iliac) were defined using imaging studies with appropriate margin expansions for CTV and PTV. Total dose was 50.4 Gy for T2, 54 Gy for T3-T4, and 45 Gy for elective lymph node regions.

\section{Toxicity}

Acute toxicity was defined as treatment effect occurring between radiation therapy start and 8 weeks after radiation treatment completion [20]. Late-developing effects were defined as occurring from 8 weeks through 1 year post treatment, the longest period of follow-up for the majority of IMRT recipients. Acute toxicities assessed were localized pain, diarrhea, fatigue, dermatitis, hematologic changes, incontinence, enterocolitis, colitis, proctitis, fistula formation, and anal/vaginal stenosis. Late toxicities included intractable diarrhea, anal/ vaginal stenosis, enterocolitis, proctitis, anal ulcers, fistula formation, wound dehiscence, and refractory pain. The Common Terminology Criteria for Adverse Events v3.0 (CTCAEv3) quantified toxicity [21].

\section{Treatment response and follow-up}

Tumor response was assessed by physical exam and follow-up imaging. Recurrence was determined clinically by physical exam. Biopsy was only performed if the lesion was clinically indeterminate. A complete response (CR) was recorded if there was no evidence of residual tumor, partial response (PR) if there was a response of at least $30 \%$ on exam or imaging compared with presentation, or progressive disease (PD), defined as an increase in tumor size on exam or imaging. Follow-up was every $3-$ 4 months for the first year, and every 4-6 months thereafter without evidence of clinical recurrence. 


\section{Results}

\section{Patient factors}

Of 165 patients, 61 (37\%) received IMRT and 104 (63\%) received 3D-CRT. Since the start of 2010, $76.7 \%$ of patients received IMRT, rising to $83.3 \%$ after 2012. Both treatment groups were demographically comparable (Table 1), and the majority had a favorable ECOG performance status of $0-1$ (85.2 and $94.3 \%$, respectively). Though HIV infection was more common in the IMRT group (13.1 vs $5.8 \%$ in $3 \mathrm{D}$ CRT), there was a comparable percentage of patients in each group receiving chronic immunosuppressive therapy (14.8\% in the IMRT group and $10.6 \%$ in the 3D-CRT group).

\section{Tumor factors}

Squamous cell carcinoma was the most common histology in both IMRT and 3D-CRT groups $(91.8 \%, 93.3 \%)$. Adenocarcinoma, cloacogenic, small cell, mucinous, and adenosquamous tumors were rarer histologies. Seventyseven percent of the tumors in both groups were T2-3 (Table 1).

\section{Chemotherapy}

One hundred fifty-two of $165(92.1 \%)$ patients received 2 cycles of concurrent infusional 5-FU-based chemotherapy. Of these 152 patients, $135(88.8 \%)$ were treated with combination 5-FU and bolus MMC according to the Nigro protocol, 46 (75.4\%) in the IMRT group, and $89(85.6 \%)$ in the 3D-CRT group.

\section{Radiation treatment}

In the IMRT group, $55.9 \%$ of patients were treated supine and $44.1 \%$ prone, while 84.8 and $15.2 \%$ of 3D-CRT patients were treated supine and prone, respectively. Patients in the IMRT group received a higher mean pelvic dose compared to 3DCRT (48.3 vs 44 Gy) with a mean tumor boost of 9.6 and 12.7 Gy, respectively. The mean total dose, number of fractions, and overall length of treatment were the same for both groups. 3D-CRT patients exhibited a wider range of treatment length compared to IMRT (25-110 vs 31-71 days) (Table 2).

\section{Clinical outcomes}

The mean and median follow-up after IMRT and 3D-CRT was 20 months (0.33-76) and 14.7 months, and 47.4 months (1125 ) and 46.1 months, respectively. In the IMRT group, rates of CR, PR, and PD were 83.6, 8.2, and 8.2\%, respectively, very similar to those among patients treated with 3D-CRT, which were $85.6,6.7$, and $7.7 \%$, respectively $(p=0.608$,
Table 1 Demographic factors $(n=165)$

Value $(\%)$

$\operatorname{IMRT}(n=61) \quad 3 \mathrm{D}-\mathrm{CRT}(n=104)$

Sex

Male

$21(34.4)$

37 (35.6)

Female

40 (65.6)

67 (64.4)

Age

Mean

Range

$58.8 \pm 10.9$

$55.9 \pm 11.5$

Performance status

ECOG 0-1

2

3

KPS $100-90$

$80-70$

$\leq 60$

BMI

$<18.5$

$18.5-24.9$

25-29.9

$\geq 30$

Smoking

Yes

Pack years (average)

HPV positive

HIV positive

Immunosuppressive therapy

Tumor type

Squamous cell

Adenocarcinoma

Other

Anal verge distance

Mean

Range

Spanning verge

T stage

1

2

3

4

$\mathrm{N}$ stage

0

38-89

26-85

$52(85.2)$

98 (94.3)

7 (11.5)

$2(1.9)$

2 (3.3)

$4(3.8)$

$38(62.3)$

68 (65.4)

$21(34.4)$

$32(30.8)$

2 (3.3)

$4(3.8)$

0 (0)

3 (2.9)

28 (45.9)

40 (38.5)

$22(36.1)$

$26(25)$

11 (18)

35 (33.7)

36 (59.0)

69 (66.3)

$26.1 \pm 21.5$

$32.7 \pm 22.0$

10 (16.4)

11 (10.6)

8 (13.1)

$6(5.8)$

9 (14.8)

11 (10.6)

56 (91.8)

97 (93.3)

3 (4.9)

$4(3.8)$

2 (3.3)

3 (2.9)

$0.44 \pm 0.99 \mathrm{~cm}$

$0.61 \pm 1.22 \mathrm{~cm}$

$0-4 \mathrm{~cm}$

0-6 cm

25 (41.0)

49 (47.1)

8 (13.1)

12 (11.5)

34 (55.7)

53 (51.0)

13 (21.3)

27 (26.0)

$6(9.8)$

12 (11.5)

23 (37.7)

59 (56.7)

14 (23.0)

19 (18.3)

13 (21.3)

16 (15.4)

11 (18.0)

$10(9.6)$

M stage

0

54 (88.5)

96 (92.3)

1

7 (11.5)

8 (7.7)

Tumor grade

1

$9(14.8)$

15 (14.4) 
Table 1 (continued)

\begin{tabular}{lll}
\hline \multirow{4}{*}{} & \multicolumn{2}{l}{ Value $(\%)$} \\
\cline { 2 - 3 } & IMRT $(n=61)$ & 3D-CRT $(n=104)$ \\
\hline 2 & $39(63.9)$ & $64(61.5)$ \\
3 & $13(21.3)$ & $25(24.0)$ \\
\hline
\end{tabular}

$I M R T$ intensity-modulated radiation therapy, 3D-CRT 3-dimentional conventional radiation therapy, ECOG Eastern Cooperative Oncology Group, KPS Karnofsky performance status

$p=0.728, p=0.729$ ). Median overall survival (OS) was not reached for the IMRT group and was 64.2 months for 3DCRT. There was no statistically significant difference in OS and event-free survival (EFS) $(p=0.971, p=0.900)$ (Fig. 1). Local recurrences were found in eight (13.1\%) and seven $(6.7 \%)$ patients in the IMRT and 3D-CRT groups, respectively $(p=0.118)$. Six $(9.8 \%)$ and 15 $(14.4 \%)$ of IMRT and 3D-CRT patients respectively recurred distantly ( $p=0.373$ ), the majority of which were to liver, lung, perirectal, and inguinal lymph nodes.

\section{Toxicity}

IMRT resulted in less acute high grade (3-4) toxicity, reaching statistical significance for pelvic pain $(p=0.033)$ and fatigue $(p=0.030)$. Decreased rates of high-grade dermatitis ( $p=0.067)$, incontinence $(p=0.094)$, and hematologic abnormalities $(p=0.239)$ resulted from IMRT as well. There were no reported instances of grade 3 fatigue or incontinence with IMRT. In both groups, grade 4 toxicity was minimal, three instances $(4.9 \%)$ in the IMRT group and seven instances $(6.8 \%)$ in the 3D-CRT group. During the 8-week period following treatment, the 3D-CRT group experienced seven instances of grade 3 toxicity, compared to one instance after IMRT. These were all similar in nature to the latedeveloping toxicities that occurred from 8 weeks post treatment through 1 year. Hematologic toxicity and dermatitis were the most prevalent high-grade acute toxicities for both IMRT and 3D-CRT (Table 3). Both groups experienced a high

Table 2 Radiation treatment regimen

\begin{tabular}{lrrrll}
\hline & \multicolumn{2}{l}{ Mean (SD) } & & \multicolumn{2}{l}{ Range } \\
\cline { 2 - 3 } \cline { 6 - 6 } & IMRT & 3D-CRT & & IMRT & 3D-CRT \\
\hline Pelvic dose & $48.3(6.2)$ & $44(6.6)$ & & $59.4-30.6$ & $63-30$ \\
Boost & $9.6(4.3)$ & $12.7(4.7)$ & & $23.4-3.6$ & $24-4$ \\
Total Gy & $53.8(4.9)$ & $54.1(7.4)$ & $62.5-32.4$ & $64-30$ \\
Fractions & $29.2(3.1)$ & $28.4(4.5)$ & & $34-18$ & $35-10$ \\
Total length (days) & $43.6(8.0)$ & $44.5(11.8)$ & & $31-71$ & $25-110$ \\
\hline
\end{tabular}

$S D$ standard deviation, Gy Gray degree of acute low-grade (1-2) toxicities, the most prevalent being grade 2 localized dermatitis. IMRT resulted in significantly less low-grade bladder/bowel incontinence compared to 3D-CRT ( $p=0.035$ ). While not statistically significant, IMRT also resulted in fewer occurrences of grade 2 localized pain, 32.8 vs $45.2 \%(p=0.115)$.

Overall, there were significantly fewer late-developing post-treatment toxicities caused by IMRT ( 4 occurrences, $6.6 \%$ of patients) within 1 year of treatment completion vs 3D-CRT (18 occurrences, $15.4 \%$ of patients) $(p=0.008)$. This trend was apparent in every toxicity category assessed except for anal ulcers and stenosis, which was reported in one and two instances, respectively, in each of the two treatment groups. Among 3D-CRT patients, five patients reported proctitis and five patients reported localized pain (Table 6).

Limiting the comparison of toxicity to patients who received MMC chemotherapy resulted in decreased rates of acute and chronic toxicity in the IMRT group comparable to those from the complete group analysis (Tables 4 and 7). IMRT continued to yield lower acute high-grade pain (2 vs 12 occurrences, 4.3 vs $13.5 \%, p=0.078$ ) and fatigue ( 0 vs 2 occurrences, 0 vs $2.2 \%, p=0.034$ ), as well as decreased rates of high-grade dermatitis (5 vs 18 occurrences, 10.9 vs $20.2 \%$, $p=0.158$ ), incontinence ( 0 vs 3 occurrences, 0 vs $3.3 \%$, $p=0.111$ ), and hematologic toxicity (6 vs 17 occurrences, 13 vs $19.1 \%, p=0.366$ ). Grade 4 toxicity remained minimal, with one less occurrence relative to the complete analysis in the 3D-CRT group. IMRT also continued to yield decreased low-grade incontinence (14 vs 42 occurrences, 30.4 vs $47.2 \%$, $p=0.059)$ and grade 2 pain (16 vs 39 occurrences, 34.8 vs $43.8 \%, p=0.309)$. Long-term toxicity results were similar to the complete analysis as well. One less instance of stenosis after IMRT and pain after 3D-CRT were recorded, totaling 6.5 and $19.1 \%$ of patients (3 vs 17 occurrences), respectively $(p=0.078)$.

Of patients who received IMRT, 27.9\% had at least one unplanned treatment break, while $41.3 \%$ in the 3D-CRT group required a treatment interruption $(p=0.89)$. Patients receiving 3D-CRT missed a mean 4.1 days, compared with 2.9 days for IMRT patients $(p=0.229)$. Of 3D-CRT recipients, $17.3 \%$ missed $\geq 10$ days, compared to $9.8 \%$ of IMRT recipients $(p=0.178)$.

\section{Discussion}

We conducted the largest single-institution study directly comparing outcomes and toxicities of patients treated with 3D-CRT vs IMRT and concurrent 5-FU/MMC for biopsyproven anal cancer. No statistical significance in OS or EFS emerged between the two groups. IMRT, however, resulted in significantly less acute and late toxicity. While the percentage of patients who required treatment breaks was similar, the 
(a) Overall survival $(p=0.971)$

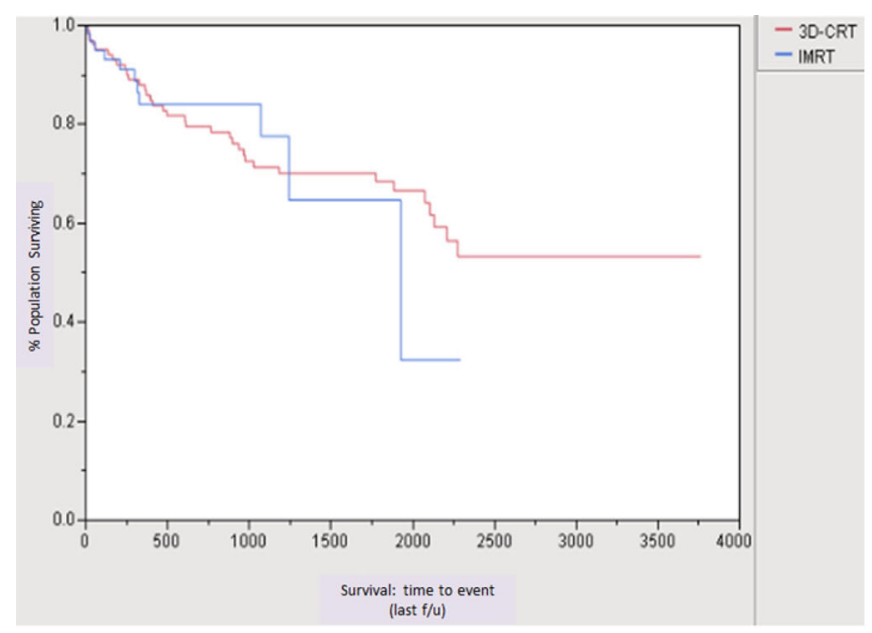

(b) Event free survival $(p=0.900)$

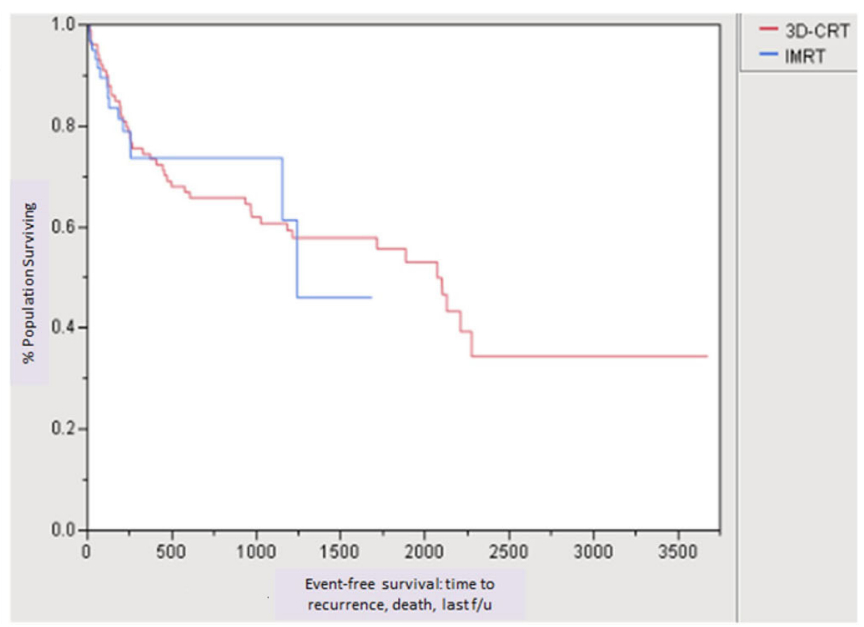

Fig. 1 Kaplan-Meier analysis of overall survival and event-free survival by treatment modality

IMRT group had shorter breaks (4.1 vs 2.9 days), as well as required fewer extensive ( $\geq 10$ days) breaks compared to the 3D-CRT group.

The relatively brief long-term follow-up time of this study likely limited the statistical significance of OS and EFS as clinical outcomes measures. However, although OS and EFS are often used to assess treatment efficacy, they may not be relevant markers in the context of anal cancer as local recurrences can be salvaged with APR, with a 75\% 5-year survival when clean margins are achieved [22]. Survival analyses, therefore, are confounded by the bias introduced by a successful salvage treatment and, as such, are largely measures of death by other causes. It is, therefore, difficult to extrapolate the degree to which reported OS and EFS advantages of IMRT are truly indicative of increased efficacy. Other, more specific end points that have been utilized in large prospective trials include toxicity, locoregional control, complete locoregional response, and progression-free survival [23].

The primary advantage of IMRT over 3D-CRT in our series was reduced acute and late toxicity, in particular high-grade toxicity (Table 3). These outcomes were notwithstanding the higher mean pelvic dose delivered with IMRT ( $48.3 \mathrm{~Gy}$ ) compared to 3D-CRT (44 Gy). While some reports, including from the RTOG, recommended a pelvic dose of 30.6 Gy for

Table 3 Acute toxicities from chemoradiation treatment

\begin{tabular}{|c|c|c|c|c|c|c|c|c|}
\hline & \multicolumn{8}{|l|}{ Value $(\%)$} \\
\hline & \multicolumn{2}{|l|}{ Grade 1} & \multicolumn{2}{|l|}{ Grade 2} & \multicolumn{2}{|l|}{ Grade 3} & \multicolumn{2}{|l|}{ Grade 4} \\
\hline & IMRT & 3D-CRT & IMRT & 3D-CRT & IMRT & 3D-CRT & IMRT & 3D-CRT \\
\hline Localized pain & $25(41.0)$ & $28(26.9)$ & $20(32.8)$ & $47(45.2)$ & $2(3.3)$ & $12(11.5)$ & $0(0)$ & $1(1.0)$ \\
\hline Diarrhea & $19(31.1)$ & $38(36.5)$ & $14(23.0)$ & $26(25)$ & $4(6.6)$ & $8(7.7)$ & $0(0)$ & $1(1.0)$ \\
\hline Fatigue & $20(32.8)$ & $32(30.8)$ & $15(24.6)$ & $23(22.1)$ & $0(0)$ & $5(4.8)$ & $0(0)$ & $0(0)$ \\
\hline Dermatitis & $16(26.2)$ & $22(21.2)$ & $37(60.7)$ & $58(55.8)$ & $5(8.2)$ & $19(18.3)$ & $0(0)$ & $0(0)$ \\
\hline Hematologic & $19(31.1)$ & $43(41.3)$ & $14(23)$ & $24(23.1)$ & $4(6.6)$ & $15(14.4)$ & $3(4.9)$ & $4(3.8)$ \\
\hline Incontinence & $12(19.7)$ & $30(28.8)$ & $5(8.2)$ & $16(15.4)$ & $0(0)$ & $2(1.9)$ & $0(0)$ & $1(1.0)$ \\
\hline Gastroenteritis $^{\mathrm{a}}$ & $0(0)$ & $0(0)$ & $0(0)$ & $0(0)$ & $1(1.0)$ & $1(1.0)$ & $0(0)$ & $0(0)$ \\
\hline Colitis & $0(0)$ & $0(0)$ & $0(0)$ & $0(0)$ & 0 & $2(1.9)$ & $0(0)$ & $0(0)$ \\
\hline Proctitis & $0(0)$ & $0(0)$ & $0(0)$ & $0(0)$ & 0 & $1(1.0)$ & $0(0)$ & $0(0)$ \\
\hline Fistula & $0(0)$ & $0(0)$ & $0(0)$ & $0(0)$ & 0 & $2(1.9)$ & $0(0)$ & $0(0)$ \\
\hline Stenosis & $0(0)$ & $0(0)$ & $0(0)$ & $0(0)$ & 0 & $1(1.0)$ & $0(0)$ & $0(0)$ \\
\hline
\end{tabular}

$I M R T$ intensity-modulated radiation therapy, 3D-CRT 3-dimensional conventional radiation therapy

${ }^{\mathrm{a}}$ From treatment completion through 8 weeks post treatment 
Table 4 Acute toxicities from chemoradiation treatment—patients who received mitomycin-C (IMRT 75.4\%; 3D-CRT 85.6\%)

\begin{tabular}{|c|c|c|c|c|c|c|c|c|}
\hline & \multicolumn{8}{|l|}{ Value (\%) } \\
\hline & \multicolumn{2}{|l|}{ Grade 1} & \multicolumn{2}{|l|}{ Grade 2} & \multicolumn{2}{|l|}{ Grade 3} & \multicolumn{2}{|l|}{ Grade 4} \\
\hline & IMRT & 3D-CRT & IMRT & 3D-CRT & IMRT & 3D-CRT & IMRT & 3D-CRT \\
\hline Localized pain & $19(41.3)$ & $26(29.2)$ & $16(34.8)$ & $39(43.8)$ & $2(4.3)$ & $11(12.4)$ & $0(0)$ & $1(1.1)$ \\
\hline Diarrhea & $15(32.6)$ & $34(38.2)$ & $8(17.4)$ & $23(25.8)$ & $4(8.7)$ & $7(7.9)$ & $0(0)$ & $0(1.1)$ \\
\hline Fatigue & $15(32.6)$ & $28(31.5)$ & $10(21.7)$ & $22(24.7)$ & $0(0)$ & $2(2.2)$ & $0(0)$ & $0(0)$ \\
\hline Dermatitis & $11(23.9)$ & $14(15.7)$ & $30(65.2)$ & $54(60.7)$ & $5(10.9)$ & $18(20.2)$ & $0(0)$ & $0(0)$ \\
\hline Hematologic & $15(32.6)$ & $36(40.4)$ & $10(21.7)$ & $22(24.7)$ & $3(6.5)$ & $13(14.6)$ & $3(6.5)$ & $4(4.5)$ \\
\hline Incontinence & $11(23.9)$ & $28(31.5)$ & $3(6.5)$ & $14(15.7)$ & $0(0)$ & $2(2.2)$ & $0(0)$ & $1(1.1)$ \\
\hline Gastroenteritis ${ }^{\mathrm{a}}$ & $0(0)$ & $0(0)$ & $0(0)$ & $0(0)$ & $1(2.2)$ & $1(1.1)$ & $0(0)$ & $0(0)$ \\
\hline Colitis & $0(0)$ & $0(0)$ & $0(0)$ & $0(0)$ & 0 & $2(2.2)$ & $0(0)$ & $0(0)$ \\
\hline Proctitis & $0(0)$ & $0(0)$ & $0(0)$ & $0(0)$ & 0 & $1(1.1)$ & $0(0)$ & $0(0)$ \\
\hline Fistula & $0(0)$ & $0(0)$ & $0(0)$ & $0(0)$ & 0 & $1(1.1)$ & $0(0)$ & $0(0)$ \\
\hline Stenosis & $0(0)$ & $0(0)$ & $0(0)$ & $0(0)$ & 0 & $1(1.1)$ & $0(0)$ & $0(0)$ \\
\hline
\end{tabular}

$I M R T$ intensity-modulated radiation therapy, $3 D$-CRT 3-dimensional conventional radiation therapy

${ }^{\mathrm{a}}$ From treatment completion through 8 weeks post treatment

conformal radiotherapy due to concerns for toxicity [24-26], doses of 40-45 Gy are commonly prescribed. The prospective trial RTOG 05-29 revealed a significant reduction in grade $\geq 3$ dermatologic and gastrointestinal and grade $\geq 2$ hematologic toxicities using IMRT to deliver 4245 Gy to the pelvis before boost [20].

The most prevalent high-grade acute toxicity in both groups was hematologic (11.5 and $18.2 \%$ for IMRT and 3DCRT, respectively), primarily leukopenia. This may have been due in part to the known immunosuppressant effects of MMC, which $84.8 \%$ of patients received, as well as inclusion of the broad pelvic bones in the radiation field [27]. IMRT, which limits the dose delivered to the greatest regions of pelvic bone marrow, had a lower rate compared with 3D-CRT. Of note, controlling for the presence of MMC resulted in nearly identical rates of toxicity compared to the whole group analysis in which fewer IMRT patients received MMC (75.4\%) than did 3D-CRT patients $(85.6 \%)$ (Table 5). Other studies found high rates of grade 3-4 hematologic toxicity, for example, $61 \%$ in RTOG 98-11 using conventional techniques [28], and 31\%

Table 5 Change in percent (\%) acute toxicity: Total group $\rightarrow$ patients who received mitomycin-C (IMRT 75.4\%; 3D-CRT 85.6\%)

\begin{tabular}{|c|c|c|c|c|c|c|c|c|}
\hline & \multicolumn{2}{|l|}{ Grade 1} & \multicolumn{2}{|l|}{ Grade 2} & \multicolumn{2}{|l|}{ Grade 3} & \multicolumn{2}{|l|}{ Grade 4} \\
\hline & IMRT & 3D-CRT & IMRT & 3D-CRT & IMRT & 3D-CRT & IMRT & 3D-CRT \\
\hline Localized pain & $41 \rightarrow 41.3$ & $26.9 \rightarrow 29.2$ & $32.8 \rightarrow 34.8$ & $45.2 \rightarrow 43.8$ & $3.3 \rightarrow 4.3$ & $11.5 \rightarrow 12.4$ & 0 & $1 \rightarrow 1.1$ \\
\hline Diarrhea & $31.1 \rightarrow 32.6$ & $36.5 \rightarrow 38.2$ & $23 \rightarrow 17.4$ & $25 \rightarrow 25.8$ & $6.6 \rightarrow 8.7$ & $7.7 \rightarrow 7.9$ & 0 & $1 \rightarrow 1.1$ \\
\hline Fatigue & $32.8 \rightarrow 32.6$ & $30.8 \rightarrow 31.5$ & $24.6 \rightarrow 21.7$ & $22.1 \rightarrow 24.7$ & 0 & $4.8 \rightarrow 2.2$ & 0 & 0 \\
\hline Dermatitis & $26.6 \rightarrow 23.9$ & $21.2 \rightarrow 15.7$ & $60.7 \rightarrow 65.2$ & $55.8 \rightarrow 60.7$ & $8.2 \rightarrow 10.9$ & $18.3 \rightarrow 20.2$ & 0 & 0 \\
\hline Hematologic & $31.1 \rightarrow 32.6$ & $41.3 \rightarrow 40.4$ & $23 \rightarrow 21.7$ & $23.1 \rightarrow 24.7$ & $6.6 \rightarrow 6.5$ & $14.4 \rightarrow 14.6$ & $4.9 \rightarrow 6.5$ & $3.8 \rightarrow 4.5$ \\
\hline Incontinence & $19.7 \rightarrow 23.9$ & $28.8 \rightarrow 31.5$ & $8.2 \rightarrow 6.5$ & $15.4 \rightarrow 15.7$ & 0 & $1.9 \rightarrow 2.2$ & 0 & $1 \rightarrow 1.1$ \\
\hline Gastroenteritis $^{\mathrm{a}}$ & 0 & 0 & 0 & 0 & $1 \rightarrow 2.2$ & $1 \rightarrow 1.1$ & 0 & 0 \\
\hline Colitis & 0 & 0 & 0 & 0 & 0 & $1.9 \rightarrow 2.2$ & 0 & 0 \\
\hline Proctitis & 0 & 0 & 0 & 0 & 0 & $1 \rightarrow 1.1$ & 0 & 0 \\
\hline Fistula & 0 & 0 & 0 & 0 & 0 & $1.9 \rightarrow 1.1$ & 0 & 0 \\
\hline Stenosis & 0 & 0 & 0 & 0 & 0 & $1 \rightarrow 1.1$ & 0 & 0 \\
\hline
\end{tabular}

$I M R T$ intensity-modulated radiation therapy, $3 D$-CRT 3-dimensional conventional radiation therapy

${ }^{\mathrm{a}}$ From treatment completion through 8 weeks post treatment 
reported by Pepek et al. with IMRT (47 patients, no direct 3DCRT comparison) [16]. Milano et al. found no benefit to IMRT (17 patients) relating to hematologic toxicity compared to 3D-CRT (7 patients) (rates not reported) [11].

Toxicity rates from both IMRT and 3D-CRT in our study were also lower than those presented in previous studies. In the 2008 report from RTOG 98-11, $48 \%$ of patients receiving 3D-CRT and 5-FU/MMC had grade 3-4 dermatologic toxicity based on the CTCAE v2.0 [28], compared with $18.3 \%$ grade 3 toxicity in our series. Kachnic et al. reported a $20 \%$ rate of grade $\geq 3$ dermatologic toxicity, as well as $22 \%$ of patients experiencing grade $\geq 3$ gastrointestinal toxicity for IMRT [29] (43 patients, no direct 3D-CRT comparison), compared with 8.2 and $6.6 \%$ in our study. Pepek et al. reported rates of $9 \%$ grade 3 and no grade 4 diarrhea, comparable to our findings [16].

IMRT reduced toxicity up to 1 year following the end of chemoradiation (Table 6). While few studies have investigated post-treatment toxicity, even fewer have reported on specific categories of long-term sequelae [13]. In our series, notable differences emerged for proctitis ( 0 vs 5$)$ and pain ( 0 vs 5$)$, with IMRT demonstrating fewer chronic toxicities in six of eight categories analyzed. Such chronic sequelae are debilitating and substantially contribute to the cost of disease management. While analyzing influences on treatment breaks and toxicity from radiation for gastrointestinal malignancies, Hill et al. reported a strong correlation between toxicity and unplanned hospital admissions [30], which inevitably entails added resources and services. In contrast, Hodges et al., based on a novel Markov decision model designed to assess both cost and quality of life discrepancies between patients with anal cancer treated with IMRT vs 3D-CRT, concluded a cost-ineffectiveness of IMRT despite its lower overall risk of acute toxicity [31].

Table 6 Late toxicities within 1 year of treatment

\begin{tabular}{lll}
\hline & \multicolumn{2}{l}{ Number of occurrences } \\
\cline { 2 - 3 } & IMRT & 3D-CRT \\
\hline Diarrhea & 1 & 2 \\
Stenosis & 2 & 2 \\
Enterocolitis & 0 & 1 \\
Proctitis & 0 & 5 \\
Anal ulcer & 1 & 1 \\
Fistula & 0 & 1 \\
Wound dehiscence & 0 & 1 \\
Pain & 0 & 5 \\
Total occurrences & 4 & 18 \\
\% of patients & 6.6 & 15.4 \\
\hline
\end{tabular}

$I M R T$ intensity-modulated radiation therapy, 3D-CRT3-dimensional conventional radiation therapy
In our analysis, there was a trend towards fewer and shorter treatment breaks for IMRT patients compared with 3D-CRT. Recent studies have suggested that treatment breaks are associated with poorer clinical outcomes: RTOG 92-08, which included a pre-specified 2-week treatment break, resulted in decreased overall, diseasefree, and colostomy-free survival compared to trials with no treatment breaks [32]. Similarly, Roohipour et al. and investigators of the ACT II trial both reported that treatment breaks contributed to worse OS, relapse-free survival, and local control [33, 34]. Other series, however, failed to show any correlation between treatment duration, breaks and outcome [12, 35]. In our series, IMRT patients experienced significantly fewer instances of high-grade toxicities, likely resulting in their tendency to need shorter interruptions. Rates of low-grade toxicity were only slightly reduced with IMRT, perhaps resulting in the comparable need for short treatment breaks (Table 7).

Studies that found a positive correlation between treatment breaks and worse outcomes have concluded that interruptions can be used as a quantitative surrogate for toxicity, based on the assumption that unplanned breaks are an opportunity to recover from an acute radiationinduced toxicity. Differences in physicians' willingness to continue treatment despite toxicities, unique institutional protocols, and vigilance in toxicity prevention, however, all contribute variability. Additionally, some institutions elect to treat over weekends, or may lack the ability to continue treatment in spite of a hospital admission or other need. These considerations weaken the ability to directly equate toxicity with treatment interruption both between and within institutions.

Table 7 Late toxicities within 1 year of treatment-patients who received mitomycin-C (IMRT 75.4\%; 3D-CRT 85.6\%)

\begin{tabular}{lll}
\hline & \multicolumn{2}{l}{ Number of occurrences } \\
\cline { 2 - 3 } & IMRT & 3D-CRT \\
\hline Diarrhea & 1 & 2 \\
Stenosis & 1 & 2 \\
Enterocolitis & 0 & 1 \\
Proctitis & 0 & 5 \\
Anal ulcer & 1 & 1 \\
Fistula & 0 & 1 \\
Wound dehiscence & 0 & 1 \\
Pain & 0 & 4 \\
Total occurrences & 3 & 17 \\
\% of patients & 6.5 & 19.1
\end{tabular}

IMRT intensity-modulated radiation therapy, 3D-CRT 3-dimensional conventional radiation therapy 
Possible limitations of our analysis include heterogeneity in the exact chemotherapy and radiation given, as well as inter-rater variability of physician-reported toxicity. Both potential biases, however, should be equally present in both groups. Furthermore, IMRT was gradually adopted as the primary treatment modality for anal cancer at our institution (2006-2012) while 3D-CRT was being utilized (2003-2012), which should have limited differences in the learning curve or level of caregiver training between treatment types. Another limitation of our study is the relatively short follow-up time of IMRT (median 14.7 months) limiting the reporting of late toxicity to 1 year post treatment. In order to perform the most robust toxicity analysis possible with the greatest number of patients, we were limited to long-term follow-up of 1 year. In the context of a comparison of chronic toxicity, however, this is a relatively short time. While a clear trend of decreased post-treatment toxicity was established, a longer follow-up comparison would likely reveal more definitive results. Additional prospective studies with longer follow-up time following IMRT would further elucidate its benefits.

\section{Conclusions}

In conclusion, IMRT for anal cancer treatment results in reduced acute and late toxicity compared to 3D-CRT. IMRT also allows for safe dose escalation with survival and recurrence outcomes similar to 3D-CRT. Patients who received IMRT required less extensive treatment breaks. A decreased rate of grade 3-4 toxicity may help reduce the need for costly therapies and services to address side effects.

\section{Compliance with ethical standards}

Funding No funding was received for this study.

Conflict of interest The authors declare that they have no conflict of interest.

Ethical approval IRB approval was obtained to perform this retrospective analysis. This article does not contain any studies with human participants or animals performed by any of the authors.

Informed consent Statement of informed consent was not applicable since the manuscript does not contain any patient data.

Open Access This article is distributed under the terms of the Creative Commons Attribution 4.0 International License (http:// creativecommons.org/licenses/by/4.0/), which permits unrestricted use, distribution, and reproduction in any medium, provided you give appropriate credit to the original author(s) and the source, provide a link to the Creative Commons license, and indicate if changes were made.

\section{References}

1. SEER Cancer Statistics Review, 1975-2013: National Cancer Institute (2015). http://seer.cancer.gov/csr/1975_2013/. Accessed 17 Apr 2017

2. Siegel R, Ward E, Brawley O, Jemal A (2011) Cancer statistics, 2011: the impact of eliminating socioeconomic and racial disparities on premature cancer deaths. CA: A Cancer J Clin 61:212-236. doi:10.3322/caac.20121

3. Martin FT, Kavanagh D, Waldron R (2009) Squamous cell carcinoma of the anal canal. Surgeon 7:232-237. doi:10.1016/S1479666X(09)80091-7

4. Klotz RG Jr, Pamukcoglu T, Souilliard DH (1967) Transitional cloacogenic carcinoma of the anal canal: clinicopathologic study of three hundred seventy-three cases. Cancer 20:1727-1745. doi: $10.1002 / 1097-0142(196710) 20: 10<1727:$ :A ID CNCR2820201024>3.0.CO;2-1

5. Ryan DP, Compton CC, Mayer RJ (2000) Carcinoma of the anal canal. N Engl J Med 342:792-800. doi:10.1056/ NEJM200003163421107

6. Mundt AJ, Lujan AE, Rotmensch J, Waggoner SE, Yamada SD, Fleming G, Roeske JC (2002) Intensity-modulated whole pelvic radiotherapy in women with gynecologic malignancies. Int $\mathrm{J}$ Radiat Oncol Biol Phys 52:1330-1337. doi:10.1016/S03603016(01)02785-7

7. Mundt AJ, Mell LK, Roeske JC (2003) Preliminary analysis of chronic gastrointestinal toxicity in gynecology patients treated with intensity-modulated whole pelvic radiation therapy. Int J Radiat Oncol Biol Phys 56:1354-1360. doi:10.1016/S0360-3016(03) 00325-0

8. Ashman JB, Zelefsky MJ, Hunt MS, Leibel SA, Fuks Z (2005) Whole pelvic radiotherapy for prostate cancer using $3 \mathrm{D}$ conformal and intensity-modulated radiotherapy. Int J Radiat Oncol Biol Phys 63:765-771. doi:10.1016/j.ijrobp.2005.02.050

9. Nutting C, Dearnaley DP, Webb S (2000) Intensity modulated radiation therapy: a clinical review. Br J Radiol 73:459-469. doi:10. 1259/bjr.73.869.10884741

10. Bazan JG, Hara W, Hsu A, Kunz PA, Ford J, Fisher GA, Welton ML, Shelton A, Kapp DS, Koong AC, Goodman KA, Chang DT (2011) Intensity-modulated radiation therapy versus conventional radiation therapy for squamous cell carcinoma of the anal canal. Cancer 117:3342-3351. doi:10.1002/cncr.25901

11. Milano MT, Jani AB, Farrey KJ, Rash C, Heimann R, Chmura SJ (2005) Intensity-modulated radiation therapy (IMRT) in the treatment of anal cancer: toxicity and clinical outcome. Int J Radiat Oncol Biol Phys 63:354-361. doi:10.1016/j.ijrobp.2005.02.030

12. Dewas CV, Maingon P, Dalban C, Petitfils A, Peignaux K, Turc G, Martin E, Khoury C, Dewas S, Crehange G (2012) Does gap-free intensity modulated chemoradiation therapy provide a greater clinical benefit than 3D conformal chemoradiation in patients with anal cancer? Radiat Oncol 7:201. doi:10.1186/1748-717X-7-201

13. Chuong MD, Freilich JM, Hoffe SE, Fulp W, Weber JM, Almhanna K, Dinwoodie W, Rao N, Meredith KL, Shridhar R (2013) Intensity-modulated radiation therapy vs. 3D conformal radiation therapy for squamous cell carcinoma of the anal canal. Gastrointest Cancer Res 6:39-45. doi:10.1016/j.ijrobp.2012.07.926

14. Koerber SA, Slynko A, Haefner MF, Krug D, Schoneweg C, Kessel K, Kopp-Schneider A, Herfarth K, Debus J, Sterzing F (2014) Efficacy and toxicity of chemoradiation in patients with anal cancer-a retrospective analysis. Radiat Oncol 9:113. doi:10.1186/ 1748-717X-9-113

15. De Francesco I, Thomas K, Wedlake L, Tate D (2016) Intensitymodulated radiotherapy and anal cancer: clinical outcome and late toxicity assessment. Clin Oncol 28:604-610. doi:10.1016/j.clon. 2016.04.039 
16. Pepek JM, Willett CG, Wu QJ, Yoo S, Clough RW, Czito BG (2010) Intensity-modulated radiation therapy for anal malignancies: a preliminary toxicity and disease outcomes analysis. Int J Radiat Oncol Biol Phys 78:1413-1419. doi:10.1016/j.ijrobp.2009.09.046

17. Salati SA, Kadi AA (2012) Anal cancer - a review. Int J Health Sci 6:206-230

18. Nigro ND, Vaitkevicius VK, Buroker T, Bradley GT, Considine B (1981) Combined therapy for cancer of the anal canal. Dis Colon Rectum 24:73-75. doi:10.1007/BF02604287

19. Burdick MJ, Stephans KL (2011) Gastrointestinal (non-esophageal) radiotherapy. In: Videtic G, Vassil A (eds) Handbook of treatment planning in radiation oncology, 1st edn. Demos Medical Publishing, New York, pp 67-84

20. Kachnic LA, Winter K, Myerson RJ, Goodyear MD, Willins J, Esthappan J, Haddock MG, Rotman M, Parikh PJ, Safran H, Willett CG (2013) RTOG 0529: a phase 2 evaluation of dosepainted intensity modulated radiation therapy in combination with 5-fluorouracil and mitomycin-C for the reduction of acute morbidity in carcinoma of the anal canal. Int J Radiat Oncol Biol Phys 86: 27-33. doi:10.1016/j.ijrobp.2012.09.023

21. Trotti A, Colevas AD, Setser A, Rusch V, Jaques D, Budach V, Langer C, Murphy B, Cumberlin R, Coleman CN, Rubin P (2003) CTCAE v3.0: development of a comprehensive grading system for the adverse effects of cancer treatment. Semin Radiat Oncol 13:176-181. doi:10.1016/S1053-4296(03)00031-6

22. Mullen JT, Rodriguez-Bigas MA, Chang GJ, Barcenas CH, Crane CH, Skibber JM, Feig BW (2007) Results of surgical salvage after failed chemoradiation therapy for epidermoid carcinoma of the anal canal. Ann Surg Oncol 14:478-483. doi:10.1245/s10434-0069221-7

23. James RD, Glynne-Jones R, Meadows HM, Cunningham D, Myint AS, Saunders MP, Maughan T, McDonald A, Essapen S, Leslie M, Falk S, Wilson C, Gollins S, Begum R, Ledermann J, Kadalayil L, Sebag-Montefiore D (2013) Mitomycin or cisplatin chemoradiation with or without maintenance chemotherapy for treatment of squamous-cell carcinoma of the anus (ACT II): a randomized, phase 3, open-label, 2 x 2 factorial trial. Lancet Oncol 14:516524. doi:10.1016/S1470-2045(13)70086-X

24. John M, Pajak T, Flam M, Hoffman J, Markoe A, Wolkov H, Paris K (1996) Dose escalation in chemoradiation for anal cancer: preliminary results of RTOG 92-08. Cancer J Sci Am 2:205-211

25. Constantinou EC, Daly W, Fung CY, Willett CG, Kaufman DS, DeLaney TF (1997) Time-dose considerations in the treatment of anal cancer. Int J Radiat Oncol Biol Phys 39:651-657. doi:10.1016/ S0360-3016(97)00329-5

26. Martenson JA, Lipsitz SR, Wagner H Jr, Kaplan EH, Otteman LA, Schuchter LM, Mansour EG, Talamonti MS, Benson AB III (1996) Initial results of a phase II trial of high dose radiation therapy, 5fluorouracil, and cisplatin for patients with anal cancer (E4292): an
Eastern Cooperative Oncology Group study. Int J Radiat Oncol Biol Phys 35:745-749. doi:10.1016/0360-3016(96)00146-0

27. Hayman JA, Callahan JW, Herschtal A, Everitt S, Binns DS, Hicks RJ, Manus MM (2011) Distribution of proliferating bone marrow in adult cancer patients determined using FLT-PET imaging. Int J Radiat Oncol Biol Phys 79:847-852. doi:10.1016/J.IJROBP.2009. 11.040

28. Ajani JA, Winter KA, Gunderson LL, Pedersen J, Benson AB III, Thomas CR Jr, Mayer RJ, Haddock MG, Rich TA, Willett C (2008) Fluorouracil, mitomycin, and radiotherapy vs fluorouracil, cisplatin, and radiotherapy for carcinoma of the anal canal: a randomized controlled trial. JAMA 299:1914-1921. doi:10.1001/jama.299.16. 1914

29. Kachnic LA, Tsai HK, Coen JJ, Blaszkowsky LS, Hartshorn K, Kwak EL, Willins JD, Ryan DP, Hong TS (2012) Dose-painted intensity-modulated radiation therapy for anal cancer: a multiinstitutional report of acute toxicity in response to therapy. Int $\mathrm{J}$ Radiat Oncol Biol Phys 82:153-158. doi:10.1016/j.ijrobp.2010. 09.030

30. Hill A, Kiss N, Hodgson B, Crowe TC, Walsh AD (2011) Associations between nutritional status, weight loss, radiotherapy treatment toxicity and treatment outcomes in gastrointestinal cancer patients. Clin Nutr 30:92-98. doi:10.1016/j.clnu.2010.07.015

31. Hodges JC, Beg MS, Das P, Meyer J (2014) Cost-effectiveness analysis of intensity modulated radiation therapy versus 3dimensional conformal radiation therapy for anal cancer. Int $\mathrm{J}$ Radiat Oncol Biol Phys 89:773-783. doi:10.1016/j.ijrobp.2014. 02.012

32. Konski A, Garcia M Jr, John M, Krieg R, Pinover W, Myerson R, Willett C (2008) Evaluation of planned treatment breaks during radiation therapy for anal cancer: update of RTOG 92-08. Int J Radiat Oncol Biol Phys 72:114-118. doi:10.1016/j.ijrobp.2007. 12.027

33. Roohipour R, Patil S, Goodman KA, Minsky BD, Wong WD, Guillem JG, Paty PB, Weiser MR, Neuman HB, Shia J, Schrab D, Temple LK (2008) Squamous-cell carcinoma of the anal canal: predictors of treatment outcome. Dis Colon Rectum 51:147-153. doi:10.1007/s10350-007-9125-Z

34. James R, Wan S, Glynne-Jones R, Sebag-Montefiore D, Kadalayil L, Northover J, Cunningham D, Meadows H, Ledermann J (2009) A randomized trial of chemoradiation using mitomycin or cisplatin, with or without maintenance cisplatin/5FU in squamous cell carcinoma of the anus (ACT II). J Clin Oncol 27:LBA4009. doi:10. 1200/jco.2009.27.18s.lba4009

35. Meyer A, Meier Zu Eissen J, Karstens JH, Bremer M (2006) Chemoradiotherapy in patients with anal cancer: impact of length of unplanned treatment interruption on outcome. Acta Oncol 45: 728-735. doi:10.1080/02841860600726729 